\title{
PROKLA-Redaktion
}

\section{Editorial: Postkoloniale Studien als kritische Sozialwissenschaft}

Ein Schwerpunktheft zu postkolonialen Studien wird vielleicht die Frage provozieren, ob man sich wirklich mit diesem Thema beschäftigen muss. Wird der Gegenstandsbereich der ehemals kolonisierten Gesellschaften nicht schon von dependenztheoretischen Ansätzen, der Weltsystemtheorie oder anderen Ansätzen der kritischen Sozialwissenschaft hinreichend erfasst? Sind die postkolonialen Studien nicht einfach nur ein neuer akademischer Trend? Die dieser PROKLAAusgabe zugrunde liegende Arbeitsthese lautet, einfach gesagt, dass dem nicht so ist. Demnach liegt die kritische Intervention postkolonialer Studien in einem - in der Form etwas angestaubten, inhaltlich aber nach wie vor hochaktuellen - Postulat der Ideologiekritik und Wissenssoziologie begründet: dass der gesellschaftliche Ort der Wissensproduktion den Inhalt beeinflusst. Ein zentraler Ausgangspunkt der postkolonialen Studien liegt mithin darin, dass die Sicht des - geografisch grob vereinfacht gesprochen - globalen Nordens auf den globalen Süden, und damit auch die Sicht der sozialwissenschaftlichen Disziplinen in ihren hegemonialen, aber auch vielen kritischen Prägungen von kolonialen Mustern gekennzeichnet ist. Es sind Muster, die „uns“ als vernünftig, zivilisiert und demokratisch konstruieren, und „die Anderen" als, unzivilisiert(wobei man das heute nicht mehr so sagt), aber jedenfalls als weniger weit fortgeschritten und eben einfach als „anders“ erscheinen lässt.
Diese Muster sind auch lange nach dem Ende formaler Kolonialherrschaft wirksam, und sie sind vielgestaltig: wir begegnen ihnen im Reisebüro, wo mit exotischen Schönheiten und wilden Stammeskriegern geworben wird, ebenso wie in Talkshow-Debatten um Kopftücher, Minarette und „den Islam“ und in sozialphilosophischen Abhandlungen, die sich eine gute Weltgesellschaft nur durch Verallgemeinerung des westlichen Gesellschaftsmodells vorstellen können, oder in politikwissenschaftlichen Analysen, die die geregelten und zivilisierten Staatenkriege Europas den durch Plünderung und brutale Gewalt an der Zivilbevölkerung geprägten „Neuen Kriegen“ der Peripherie gegenüberstellen - und dabei stillschweigend nicht nur die Gräuel des Zweiten Weltkriegs, sondern auch die Massaker des Kolonialismus unter den Tisch fallen lassen. Die kritische Sozialwissenschaft ist ebenfalls nicht ganz frei von solchen Mustern: Auschwitz als „Zivilisationsbruch" $\mathrm{zu}$ bezeichnen ist insofern problematisch, als damit eine bestimmte Zivilisation vorher als intakt und eben „zivilisiert“ angesehen wird, ungeachtet der im kolonialen Kontext begangenen Völkermorde.

Postkoloniale Studien befassen sich mit diesen kolonialen Mustern, mit den Nachwirkungen des Kolonialismus in ehemals kolonisierten und ehemals kolonisierenden Gesellschaften oder, abstrakter formuliert: mit der Analyse und Kritik von Herrschaftsverhältnissen im weiteren 
Zusammenhang mit dem historischen Phänomen des Kolonialismus. Dipesh Chakrabarty hat einen Kernbestandteil des Projekts der postkolonialen Studien beschrieben als die „Provinzialisierung Europas“: eine Welt zu denken, in der Europa (und seine Siedlerkolonien in Nordamerika) nicht mehr selbstverständlicher Ausgangspunkt der Geschichtsschreibung und Theoriebildung ist - und in der eurozentrische Kategorien nicht als universeller Maßstab gelten. Dies gilt gerade auch für die Sozialwissenschaften, in denen allzu oft die Gesellschaften Westeuropas und Nordamerikas die Norm und so den zentralen Gegenstandsbereich bilden, während periphere Gesellschaften institutionell und intellektuell marginalisierten „Unterabteilungen“ wie Entwicklungssoziologie oder Ethnologie zugeschoben werden.

Über diesen vagen gemeinsamen Nenner hinaus sind in den postkolonialen Studien durchaus heterogene Ansätze aus unterschiedlichen Disziplinen zu verzeichnen. Selbst wenn die oft als Ikonen der postkolonialen Studien genannten AutorInnen Edward Said, Gayatri Spivak und Homi Bhabha aus den Literaturwissenschaften kommen, hat die postkoloniale Perspektive über die Kultur- und Geschichtswissenschaften auch die Sozialwissenschaften erreicht. Im deutschsprachigen Raum fristen die postkolonialen Studien gerade in den Sozialwissenschaften jedoch noch ein Schattendasein, aus dem sie bestenfalls allmählich ausbrechen.

Dies ist bedauerlich. Denn wenn Said die jahrhundertelange Kontinuität der Stereotypen herausarbeitet, mit denen im Westen ein bestimmtes Bild des Orients konstruiert wurde, wenn Spivak unter Rückgriff auf poststrukturalistische, marxistische und feministische Positionen die Fallstricke aufklärerischen Handelns, die Notwendigkeit von Ideologiekritik und die (Un-) Möglichkeit einer Selbstrepräsentation der Subalternen diskutiert, oder wenn Bhabha aufzeigt, wie koloniale Diskurse unerwünschte Ambivalenzen, Nebeneffekte und Hybriditäten hervorbringen und so die Herrschaft, die sie absichern sollen, gleichzeitig auch unterminieren, dann ließen sich diese Thesen durchaus produktiv auf Gegenstandsbereiche der Sozialwissenschaften anwenden. Dies gilt für die Analyse der Prozesse indigener Selbstorganisation und sozialer Bewegungen (siehe die Beiträge von Tanja Ernst und Maria Framke/ Jana Tschurenev) ebenso wie für den Bereich der Migration und Integration (dazu die Aufsätze von Petra Neuhold/ Paul Scheibelhofer und Stefanie Kron) und für allgemeinere herrschaftssoziologische Betrachtungen der Nord-Süd Beziehungen, wie sie in dem Beitrag von Aníbal Quijano unternommen werden. (Entwicklungstheorie und Entwicklungspolitik bleiben an dieser Stelle ausgeklammert, postkoloniale Debatten zu diesem Themenbereich werden in einem Schwerpunktheft der Zeitschrift Peripherie behandelt, das Ende 2010 erscheinen wird.) Der PROKLA-Heftschwerpunkt mit dem programmatischen Titel „Postkoloniale Studien als kritische Sozialwissenschaft" soll einen Beitrag dazu leisten, an dem kritisierten Zustand etwas zu ändern. In diesem Sinne geht es darum, eine breitere Auseinandersetzung mit postkolonialen Studien zu befördern, und sie als dezidiert kritische Perspektive weiter zu entwickeln. Nun kann es im Sinne der kritischen Haltung, die postkoloniale Studien gegenüber den hegemonialen Disziplinen einnehmen, gerade nicht darum gehen, diese in einen wie auch immer gearteten sozialwissenschaftlichen Kanon, „kritisch“ oder nicht, einzuverleiben. Wie María do Mar Castro Varela und Nikita Dhawan unlängst deutlich gemacht haben, handelt es sich bei postkolonialen Studien vielmehr um eine ,antidisziplinäre Intervention“. Als solche widmet sie sich unter anderem einer kritischen Analyse dessen, welche Rolle wis- 
senschaftliche Disziplinen dabei gespielt haben und weiterhin spielen, (post-)koloniale Herrschaftsstrukturen zu reproduzieren.

Daher werden die folgenden Beiträge nicht nur empirisch den Blick auf fortbestehende post- und neokoloniale Strukturen von Macht und Herrschaft für die Analyse der Gegenwart in Zentren und Peripherien lenken, und die Relevanz der Analyse dieser Strukturen für emanzipative politische Prozesse deutlich machen. Vielmehr werden sie auch erkenntnistheoretische, methodologische, und normative Fundamente kritischer sozialwissenschaftlicher Ansätze einer (Selbst-)Überprüfung unterziehen. Letztlich werden sie in vielfacher Weise aufzeigen, dass kritische Debatten und Forschungsstränge nur auf eigene Kosten - sei es in der Qualität der Theoriebildung und der empirischen Analysen, oder in uneingelösten normativen Ansprüchen - die Interventionen postkolonialer Studien ignorieren können.

Der Artikel von Joshua Kwesi Aikins und Hannah Franzki bereitet hierfür die Grundlage, indem er einen systematischen Überblick über die postkolonialen Studien gibt. Daneben werden die zahlreichen Anschlüsse diskutiert, die postkoloniale Perspektiven zu unterschiedlichsten Strängen kritischer sozialwissenschaftlicher Theoriebildung und Forschungspraxis aufweisen. Dabei qualifizieren die AutorInnen die häufig geäußerte Kritik, postkoloniale Studien vernachlässigten materielle Dimensionen der von ihnen analysierten Zustände zugunsten von Diskursen, Repräsentationen und Identitätskonstruktionen, als Kritik an nur einem Strang postkolonialer Forschung. Als Beleg für die diesbezügliche Vielfalt postkolonialer Ansätze kann der Artikel von Aníbal Quijano gelten. Er baut auf eine lateinamerikanische Tradition postkolonialer Theoriebildung auf, die sich in kritisch-konstruktiver Auseinandersetzung mit materialistisch geprägten An- sätzen, insbesondere der Dependenztheorie und Wallersteins Weltsystemtheorie entwickelt hat. Aus eben dieser Tradition schöpft auch Tanja Ernst für ihre Analyse der gegenwärtigen Bestrebungen zu einer Dekolonisierung des liberalen bolivianischen Demokratiemodells. Sie diskutiert nicht nur die Bedeutung indigener Autonomie und Demokratiekonzepte in einer gegenwärtigen postkolonialen $\mathrm{Ge}$ sellschaft, sondern zeigt auch auf, wie produktiv der Dialog über Wissensformationen hinweg für die Reflexion ihrer eigenen Position als Forscherin ist. Maria Framke und Jana Tschurenev skizzieren eine postkoloniale Perspektive auf den Faschismusbegriff, die auf die Dezentrierung der europäischen Faschismuserfahrung abzielt. Einerseits analysieren sie die zeitgenössische Rezeption vor allem des italienischen Faschismus in Indien, wobei deutlich wird, dass trotz einer bisweilen anzutreffenden Gleichsetzung von Imperialismus und Faschismus antikoloniale Bewegungen nicht immer frei waren von reaktionären Tendenzen. Andererseits diskutieren sie die Frage, inwiefern die hindunationalistische Sangh Parivar Bewegung als faschistisch $\mathrm{zu}$ bezeichnen ist. Dabei weisen sie darauf hin, dass im indischen Kontext postkoloniale Eurozentrismuskritik auch in neokonservative Diskurse einfließt. Den Blick zurück auf den Westen lenken dann Petra Neuhold und Paul Scheibelhofer, indem sie die eurozentrischen Implikationen liberaler Multikulturalismuskonzepte herausarbeiten. In den mit diesen verknüpften Einwanderungs- und Integrationspolitiken dient die Evaluierung von Menschen aus „fremden Kulturen und Völkern“ der Schaffung eines differenzierten Systems der Disziplinierung und abgestuften Entrechtung von MigrantInnen. Dem methodologischen Nationalismus dieser Konzepte gegenüber werfen sie die Frage nach einem „Multikulturalismus von unten“ auf. Sook-Young Ahn und Ralf Havertz erkunden, inwiefern sich koloniale und eu- 
rozentrische Muster in einem außereuropäischen Kontext wieder finden lassen: im Verhältnis zwischen Südkorea und Japan. An historischen und vor allem literarischen Beispielen arbeiten sie die Legitimationsmechanismen der japanischen Überlegenheitsideologie und ihrer koreanischen AnhängerInnen heraus, die u.a. einen Rassismus ohne „Rassenunterschiede" und die Vorstellung eines „gemeinsamen Volkskörpers" beinhaltet. Einen Beitrag zur theoretischen und methodischen Debatte in den postkolonialen Studien leistet schließlich Stefanie Kron, insbesondere im Hinblick auf die Konzepte der Intersektionalität und des border feminism. Empirisch zeigt sie, dass in Bezug auf die Rückkehrmigration guatemaltekischer Kriegsflüchtlinge die gängigen Interpretationsmuster $\mathrm{zu}$ eindimensional sind und die politischen Subjektivitäten durch die Überschreitung nicht nur der Staatsgrenzen (borders), sondern auch der „symbolischen Grenzen von race, class und gender" (boundaries) geprägt wurden.

Der Schwerpunkt des vorliegenden Heftes wurde maßgeblich von unseren GastredakteurInnen Matthias Ebenau, Aram Ziai, Hannah Franzki und Joshua Kwesi Aikins gestaltet. Wir bedanken uns ganz herzlich für ihr umfangreiches Engagement.

$$
* * *
$$

An dieser Stelle auch noch ein verspätetes, ganz herzliches Dankeschön an Stefan Schmalz: Er hatte als Gastredakteur mit vielen Ideen und viel persönlichem Einsatz unser letztes Heft, PROKLA 157 Der blutige Ernst: Krise und Politik, betreut. Im Stress der Endredaktion dieses Heftes ist dann allerdings die Danksagung an ihn unter den Tisch gefallen.

\section{In eigener Sache}

Seit Dezember 2009 gibt es die PROKLA-Homepage www.prokla.de in neuer Gestalt und auch mit einem neuen Service für unsere LeserInnen: Von PROKLA Nr. 1 aus dem Jahr 1971 (damals noch „Probleme des Klassenkampfs“) bis einschließlich PROKLA Nr. 149 vom Dezember 2007 stehen alle Hefte, sowie die Sonderhefte, die in den frühen 1970er Jahren erschienen sind, als Volltext kostenlos zum Download bereit. Dass das alles so übersichtlich aussieht und leicht zu handhaben ist, war nur möglich durch einen erheblichen Arbeitseinsatz. Dafür bedanken wir uns ganz herzlich bei Markus Euskirchen.

Eine weniger gute Nachricht für unsere LeserInnen ist die Preiserhöhung, die in diesem Jahr leider unumgänglich wurde. Trotz steigender Druck- und Vertriebskosten blieb der Verkaufspreis der PROKLA über Jahre hinweg konstant: Der Abopreis wurde zum letzten Mal im Jahr 1998 erhöht, der Einzelverkaufspreis im Jahr 2006. Ab diesem Jahr beträgt der Einzelverkaufspreis 14 Euro (statt bisher 12 Euro) und der Abopreis für ein Jahr 38 Euro (statt 33 Euro) plus Porto. Ein Jahresabo mit vier Heften kostet also weniger als drei Hefte im Einzelverkauf vielleicht ist das für einige LeserInnen ein zusätzliches Argument die PROKLA zu abonnieren.

Die Existenz der PROKLA wird einerseits durch eine möglichst große Zahl von Abonnements gesichert, andererseits durch die finanzielle und ideelle Unterstützung der „Vereinigung zur Kritik der politischen Ökonomie e.V.“, die die PROKLA herausgibt. Die Mitgliederversammlung der „Vereinigung“ trifft sich einmal im Jahr, um den Redaktionsbeirat und die Redaktion zu wählen und um die Schwerpunktthemen des folgenden Jahres zu debattieren. Die Mitgliedsbeiträge werden zur Unterstützung der Redaktionsarbeit verwendet. $\mathrm{Da}$ die PRO- 
KLA und die Vereinsarbeit der „Volksbildung" dienen, wir wollen schließlich die Kritik der vielfältigen Herrschaftsund Ausbeutungsverhältnisse auf solider wissenschaftlicher Grundlage, aber zugleich gut verständlich unters Volk bringen, ist die „Vereinigung“" schon seit vielen Jahren als gemeinnützig anerkannt, Spenden und Mitgliedsbeiträge können daher steuerlich abgesetzt werden. Die nächste Mitgliederversammlung findet am Samstag, den 24. April 2010 in Berlin statt. Wer mehr über die „Vereinigung“ erfahren möchte oder Interesse an einer Mitgliedschaft hat, kann gerne an redaktion@prokla.de schreiben und mehr Informationen dazu erhalten.

\section{PROKLA 159: Marx! (Juni 2010)}

Mit der eskalierenden Finanzkrise hat es auch Marx seit einer Weile wieder in die Spalten großer Tageszeitungen und Journale geschafft. Kaum verwunderlich, erklärte der ökonomische Mainstream doch seit Jahren, dass es zu größeren Krisen nicht mehr kommen würde. Allenthalben wird jetzt beklagt, dass niemand die Krise vorausgesehen habe - bis auf Marx und dementsprechend wächst auch das Interesse an seinen Theorien. Die mediale Beschäftigung mit Marx läuft allerdings auf seine Neutralisierung hinaus: trotz einiger Einsichten würden seine Theorien für die Gegenwart nichts taugen, so die meistens verkündete. Demgegenüber geht es uns gerade darum, die gegenwärtigen Entwicklungen mit Hilfe der Marxschen Theorie zu begreifen - was nicht ausschließt auch die Schwächen, Leerstellen oder Sackgassen dieser Theorie und mancher der von ihr angeregten Debatten klar zu benennen, aber auch die Mythen über die Marxsche Theorie, die nach wie vor im Umlauf sind.

In dem PROKLA-Heft soll es aber nicht allein um an Marx orientierte Krisentheorien gehen, wie sie jetzt wieder verstärkt diskutiert werden. Weit darüber hinausgehend liefert Marx die Grundelemente für eine kritische Theorie von Gesellschaft und Geschichte. Werden diese Elemente von ökonomistischen und klassenreduktionistischen Verkürzungen befreit, bieten sie fruchtbare Anschlusspunkte an andere kritische Diskurse, wie sie in feministischen oder postkolonialen Ansätzen formuliert werden. Zweck dieser analytischen Anstrengungen ist nicht bloß die Einsicht in das Funktionieren der gegenwärtigen Gesellschaft, sondern die gesellschaftliche Emanzipation. Doch wie kann heute eine an Marx anschließende Emanzipationsperspektive aussehen? Und auf welche Weise kann die angestrebte Emanzipation erreicht werden? 